\title{
Acknowledgment to reviewers who served in 2014
}

(c) The Japanese Society for Hygiene 2014

We highly appreciate a kind contribution of the following individuals who served as a reviewer for articles submitted to Environmental Health and Preventive Medicine in 2014.

Takemi Otsuki

Editor-in-Chief

Babazono, Akira (Fukuoka)

Ezura, Yoichi (Tokyo)

Fujishiro, Hitomi (Tokushima)

Fukuda, Yoshiharu (Ube)

Fukunaga, Ichiro (Kochi)

Furuya, Hiroyuki (Isehara)

Hamazaki, Kei (Toyama)

Harada, Kouji (Kyoto)

Hida, Kazuyuki (Okayama)

Hiraku, Yusuke (Tsu)

Hirano, Seishiro (Tsukuba)

Hisanaga, Naomi (Komaki)

Hiura, Mizue (Kumamoto)

Horiguchi, Hyogo (Sagamihara)

Horiuchi, Masahisa (Kagoshima)

Ichihara, Gaku (Nagoya)

Inadera, Hidekuni (Toyama)

Ishikawa, Hirofumi (Okayama)

Iwata, Noboru (Higashihiroshima)

Izawa, Tetsuya (Kyotanabe)

Kameo, Satomi (Maebashi)

Kamide, Kei (Suita)

Kamijima, Michihiro (Nagoya)

Kanamori, Masao (Otsu)

Kashihara, Naoki (Kurashiki)

Kawai, Kazuaki (Kitakyuhsu)

Kayaba, Kazunori (Koshigaya)

Kido, Teruhiko (Kanazawa)
Kobayashi, Nobumichi (Sapporo)

Koizumi, Akio (Kyoto)

Konishi, Shoko (Tokyo)

Koyama, Hiroshi (Maebashi)

Kumae, Takashi (Tokyo)

Kumagai, Yoshito (Tsukuba)

Kunugita, Naoki (Wako)

Mamoru, Hirata (Amagasaki)

Matsumura, Yasuhiro (Chigasaki)

Michikawa, Takehiro (Tsukuba)

Min, Kyong-Son (Tondabayashi)

Miyai, Nobuyuki (Wakayama)

Miyao, Masaru (Nagoya)

Miyatake, Nobuyuki (Miki-cho)

Mogi, Masaki (Toon)

Morioka, Ikuharu (Wakayama)

Morita, Emi (Nagoya)

Morita, Manabu (Okayama)

Moriyama, Masaki (Fukuoka)

Murata, Mariko (Tsu)

Nagaya, Teruo (Nagoya)

Nakai, Kunihiko (Sendai)

Nakamura, Hiroyuki (Kanazawa)

Nakamura, Keiko (Tokyo)

Nakaya, Naoki (Sendai)

Nishimura, Yasumitsu (Kurashiki)

Nishiyama, Midori (Mibu)

Nomura, Kyoko (Tokyo)
Norio, Sugawara (Hirosaki)

Ogawa, Yusuke (Kyoto)

Ohgami, Nobutaka (Kasugai)

Okubo, Yasushi (Tokyo)

Okuda, Masayuki (Yamaguchi)

Onodera, Sho (Kurashiki)

Satoh, Minoru (Kitakyuhsu)

Satoh, Minoru (Kurashiki)

Sozu, Takashi (Kyoto)

Sugisaki, Koshu (Niigata)

Suna, Shigeru (Takamatsu)

Suwazono, Yasushi (Chiba)

Suzuki, Kohta (Yamanashi)

Takebayashi, Toru (Tokyo)

Tatsuta, Nozomi (Sendai)

Tomita, Masafumi (Kurashiki)

Ueda, Kayo (Kyoto)

Ueji, Masaru (Mito)

Wada, Yasuhiko (Kochi)

Wakabayashi, Ichiro (Nishinomiya)

Wang, Da-Hong (Okayama)

Yamane, Kunikazu (Kurashiki)

Yano, Hiromi (Kurashiki)

Yonemoto, Junzou (Tsukuba)

Yoshida, Minoru (Hachinohe)

Yoshimasu, Kouichi (Wakayama)

Yoshitome, Kei (Kurashiki) 